\title{
PROGRAMA DE CONSERVACIÓN DEL GOCHU ASTUR-CELTA: CREACIÓN DE UN BANCO DE GERMOPLASMA
}

\author{
ENDANGERED GOCHU ASTUR-CELTA GERMPLASM BANK
}

\author{
Tamargo, C. ${ }^{1 *}$, Rodríguez, A. ${ }^{1}$, Fernández, A. ${ }^{1}$, Merino, M.J. ${ }^{1}$, Reyes, M.J. ${ }^{1}$, Benito, ${ } . M^{2}{ }^{2}$ \\ e Hidalgo, C.O. ${ }^{1}$
}

\begin{abstract}
${ }^{1}$ Área de Selección y Reproducción Animal. SERIDA. Centro de Biotecnología Animal. Gijón. España. ctamargo@serida.org

²Dirección General de Ganadería y Agroalimentación. Oviedo. España.
\end{abstract}

\section{PALABRAS CLAVE ADICIONALES}

Razas autóctonas. Verraco. Espermatozoide. Crioconservación.

\section{RESUMEN}

El objetivo de este trabajo fue el estudio de la posibilidad de criopreservar semen de verracos de la raza porcina autóctona Gochu Astur-Celta, que en la actualidad se encuentra en peligro de extinción, como herramienta en los planes de recuperación de la raza. Las dosis seminales obtenidas a partir de los eyaculados recogidos fueron envasadas, sometidas a congelación y posteriormente almacenadas en nitrógeno líquido, analizándose los siguientes parámetros: motilidad y calidad del movimiento, integridad funcional de la membrana, morfología y estado del acrosoma. Los resultados obtenidos muestran que es necesario seguir trabajando en la mejora de la calidad del semen congelado-descongelado para que esta técnica pueda contribuir a la conservación de la biodiversidad.

\section{SUMMARY}

The use of sperm cryoconservation technique could help to the preservation of genetic material for long term. This work was carried out in order to develop preliminary studies regarding boar sperm cryopreservation in the autochthonous endagered pig breed Gochu Astur-Celta. The seminal doses obtained from ejaculates of these boars, were cryopreserved and then stored in liquid nitrogen. Semen analysis were carried out on every sample, and the parameters studied

Presentado al Congreso SERGA (2010, Asturias).

\section{Additional KEYWORDS}

Local breeds. Boar. Spermatozoa. Cryopreservation.

were: sperm motility, movement quality, normal apical ridge (NAR), morphology and functional membrane integrity (hyposmotic swelling test). The results showed that further studies are needed to improve the quality of frozen-thawed sperm to make this technique a useful tool in the field of biodiversity preservation.

\section{INTRODUCCIÓN}

La conservación ex situ es considerada como una actividad esencial y complementaria a la conservación in situ de los recursos genéticos de animales de granja (FAO, 1988; ERPF, 2003). Desde el Servicio Regional de Investigación y Desarrollo Agroalimentario (SERIDA), se ha venido trabajando en la conservación de recursos genéticos de las razas autóctonas, siendo el objetivo de este trabajo la puesta a punto de las técnicas para la obtención y congelación de semen del Gochu Astur-Celta, ya que el semen criopreservado es una excelente manera de almacenar material genético valioso (Johnson et al., 2000).

\section{MATERIAL Y MÉTODOS}

Los machos donantes (7-24 meses de edad) fueron seleccionados por motivos 
genéticos y morfológicos por la Asociación de Criadores entre los animales del núcleo de multiplicación. Los eyaculados ( $\mathrm{N}=109)$ de los 6 verracos objeto de estudio fueron recogidos dos días por semana mediante mano enguantada (Hernández, 1976), diluidos (1:1, vol/vol) en Beltsville Thawing Solution (BTS) y se evaluaron las características seminales convencionales. El volumen se determinó en una probeta graduada y la concentración espermática mediante un espectrofotómetro (Acuccell, IMV Technologies, Francia); el porcentaje de espermatozoides móviles se evaluó de manera subjetiva (microscopio de contraste de fases, 200X) y objetiva (método computerizado de análisis de imagen) en una platina a $39^{\circ} \mathrm{C}$ entre un porta y un cubre a la misma temperatura y la calidad del movimiento en una escala de 0 a 5 ( 0 ausencia de movimiento y 5 movimiento rápido, progresivo y lineal; Martín-Rillo et al., 1996). La morfología espermática y del acrosoma se determinó en muestras húmedas fijadas con glutaraldehido al 2 p. 100 que se observaron en el microscopio óptico de contraste de fases a 400x y 1000x aumentos en inmersión, respectivamente (Pursel y Johnson, 1975). Se evaluaron un total de 200 espermatozoides por preparación. La integridad funcional de la membrana plasmática se determinó mediante el test de endósmosis, sometiendo a los espermatozoides a una solución hiposmótica y evaluando en 200 de ellos el porcentaje de colas enrolladas (endósmosis positiva, membrana funcional).

Para la criopreservación de dosis seminales se descendió la temperatura del semen a $17^{\circ} \mathrm{C}$ y se centrifugó, eliminando el sobrenadante. Se le añadió el medio de dilución LEY (a base de yema de huevo, lactosa y antibióticos) y se enfrió a $5^{\circ} \mathrm{C}\left(0,1^{\circ} \mathrm{C} / \mathrm{min}\right)$, adicionando entonces el diluyente de congelación LEYGO (LEY con 9\% de glicerol y $1,5 \%$ de Equex Stem $\left.{ }^{\circledR}\right)$. El semen se envasó en pajuelas de $0,5 \mathrm{ml}$ con 500 millones de espermatozoides por dosis, que fueron congeladas en vapores de nitrógeno y sumergidas en nitrógeno líquido, donde quedaron almacenadas. Para su evaluación postdescongelación, dos dosis por lote se sumergieron en un baño a $50^{\circ} \mathrm{C}$ durante 12 segundos (Thilmant, 1998).

\section{RESULTADOSYDISCUSIÓN}

El entrenamiento de los verracos para la obtención de semen con destino al BRZ tuvo un $87,5 \%$ de éxito (se consiguió recuperar semen de 6 de los 7 verracos cedidos); se recogió semen en 122 sesiones de las 153 sesiones totales (cerca del $80 \%$ de éxito en la recogida), procesándose para su congelación el 86\% de los eyaculados obtenidos.

En la tabla I se recogen los valores medios y el error estándar de los diferentes parámetros seminales estudiados. Los valores obtenidos no difieren de los que otros autores encontraron para los verracos de raza Chato Murciano (Peinado et al., 1998a y b) e Ibérico (Pérez-Marcos et al., 1987). La motilidad postdescongelación está próxima al 33 p.100, muy inferior a los valores medios de semen fresco. Sin embargo, la calidad del movimiento se mantenía en unos niveles aceptables (entre 3 y 4), lo que indica que

Tabla I. Características seminales de los eyaculados obtenidos de 6 verracos de raza Gochu Astur-Celta. (Sperm quality parameters of ejaculates obtained from 7 Gochu Astur-Celta boars).

Volumen $(\mathrm{ml})$

Concentración $\left(10^{6} \mathrm{spz} / \mathrm{ml}\right)$

Motilidad total fresco (\%)

Motilidad total postdescongelación (\%)

Endósmosis (\%)
$82,54 \pm 4,02$

$560,71 \pm 22,45$

$85 \pm 1,02$

$32,76 \pm 1,39$

$89,10 \pm 0,66$
Anomalías cabeza (\%)

Gota citoplasmática (\%)

Anomalías tracto intermedio (\%)

Anomalías cola

Acrosomas normales (\%)
$1,22 \pm 0,11$

$2,8 \pm 0,4$

$0,64 \pm 0,09$

$2,81 \pm 0,29$

$98,94 \pm 0,22$

Archivos de zootecnia vol. 60, núm. 231, p. 366. 
aunque el proceso de congelación daña a un número considerable de células espermáticas, las que consiguen sobrevivir mantienen una funcionalidad similar a la del semen fresco (Gadea, 1997).

La producción de espermatozoides y su respuesta a la criopreservación en la especie porcina es muy variable, debiendo conocerse los factores extrínsecos e intrínsecos al individuo que afectan dicha variabilidad, para poder mejorar la eficiencia productiva del semen conservado (Hernández, 2007). La técnica de congelación basada en el uso de yema de huevo y glicerol como agentes crioprotectores descrita por Westendorf et al. (1975), a los que se añade una elevada concentración de azúcares y un agente detergente (Orvus et paste), sigue siendo la más utilizada (Gadea, 2003) y los cambios introducidos en los protocolos de congelación no lo han modificado sustancialmente.

\section{BIBLIOGRAFÍA}

Delgado, J.V., Cabello, A., Sereno, J.R.B., Barba, C. y Sereno, F.P.S. 2000. Programa de conservación ex situ de las variedades de cerdo ibérico de la provincia de Córdoba. Arch. Zootec., 50: 139-144.

ERFP. 2003. Guidelines for the constitution of animal cryopreservation programmes for farm animals. In: Hiemstra S.J. (Ed.). European Regional Point on Animal Genetic Resources. Holanda.

Gadea, J. 1997. Predicción de la fertilidad in vivo de los eyaculados de verraco mediante parámetros rutinarios de contrastación seminal, pruebas bioquímicas y el test homólogo de penetración in vitro. Tesis doctoral Universidad de Murcia.

Gadea, J. 2003. Los diluyentes en la inseminación artificial porcina. Revisión. Spanish J. Agric. Res., 1: 17-27.

Hernández, J.J. 1976. Estudio comparativo entre la vagina artificial y mano enguantada para recolección de semen porcino. Rev. Cubana Reprod. Anim., 2: 65-70.

Hernández, M. 2007. Criopreservación espermática en la especie porcina. Variabilidad indivi-
Las posibilidades de congelación de semen porcino permite disponer de gametos conservados durante largos periodos de tiempo y el máximo aprovechamiento de estos animales de alto valor genético, siendo usada en los programas de conservación ex situ con éxito (Delgado et al., 2000; Poto et al., 2000). Los resultados obtenidos en este trabajo indican la posibilidad de que el semen del Gochu Astur-Celta pueda sobrevivir la congelación y descongelación con suficiente calidad para ser usado en conservación y mejora genética, siendo necesaria la realización de estudios complementarios.

\section{AGRADECIMIENTOS}

Este trabajo se ha desarrollado en colaboración con la Asociación de Criadores del Gochu Astur-Celta y financiado por la Consejería de Medio Rural y Pesca del Principado de Asturias y FEDER.

dual. Tesis doctoral. Universidad de Murcia.

Johnson, L.A., Weitze, K.F., Fiser, P. and Maxwell, W.M.C. 2000. Storage of boar semen. Anim. Reprod. Sci., 62: 143-172.

Martín-Rillo, S., Martínez, E., García-Artiga, C. and De Alba, C. 1996. Boar semen evaluation in practice. Reprod. Domest. Anim., 35: 519-526.

FAO. 1998. Segundo documento de líneas directrices para la elaboración de planes nacionales de gestión de los recursos genéticos de animales de granja. Gestión de pequeñas poblaciones en peligro. FAO. Roma.

Peinado, B., Poto, A., Gadea, J. y Ruiz, S. 1998a. Estudios preliminares de la criopreservación de espermatozoides porcinos de raza Chato Murciano. Arch. Zootec., 47: 305-310.

Peinado, B., Poto, A., Lobera, J.B., Martín, J. y Fernández, A. 1998b. Calidad seminal de los eyaculados de verraco de raza Chato Murciano. Arch. Zootec., 47: 311-317.

Pérez-Marcos, C., Martín Rillo, S., Sebastián, J.J. y Sánchez, R. 1987. Efecto de las distintas fracciones de esperma sobre la conservación de semen refrigerado en verracos de raza 
Ibérica. Med. Vet., 4: 605-610.

Poto, A., Peinado, B., Barba, C. y Delgado, J.V. 2000. Congelación de semen porcino de razas autóctonas en peligro de extinción. Influencia de la metodología en bancos de germoplasma para pequeñas poblaciones. Arch. Zootec., 49: 493-496.

Pursel, V.G. and Johnson, L.A. 1975. Glutaraldehyde fixation of boar spermatozoa for acrosome evaluation. Theriogenology, 1: 63-68.

Thilmant, T. 1998. Cryopreservation of boar semen in $0,5 \mathrm{ml}$ french straws. $10^{\text {th }}$ Meeting of A.I. Bruges. Belgium. pp. 1-15.

Westendorf, P., Ritcher, L. and Treu, H. 1975. Deep freezing of boar semen. Insemination experiments using the Hulserberger pailleten technique. Dtsch. Tieraztl. Wochenschr., 82: 261-300. 